Over the last year, the Planning Committee of the Board of Directors has been leading a strategic meetings initiative to develop our 10-year vision for the future.

\title{
Creating the future of MRS meetings
}

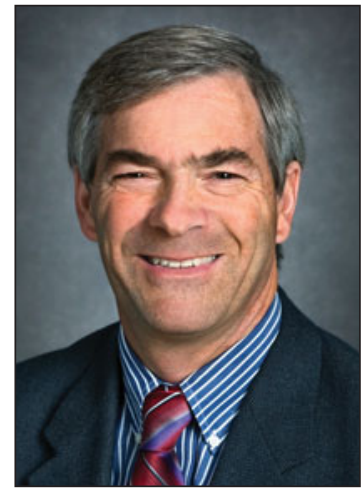

Jim De Yoreo 2011 MRS President

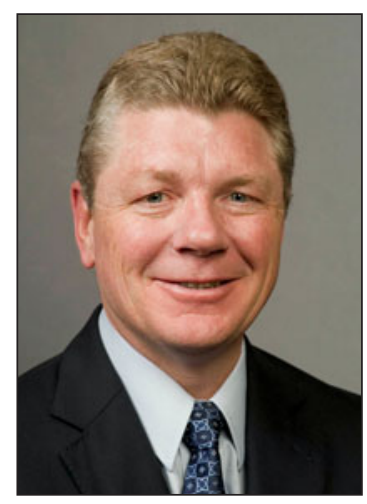

Duane Dimos
Chair, Planning Committee

For most of its history, the Materials Research Society has organized and operated its two annual meetings around a single model that emphasized, above all else, technical quality. That model has served our community well and has, arguably, been the singular source of our continued growth as a professional society. Indeed, to the materials community at large, these two high-quality technical meetings have traditionally been synonymous with "MRS." They have served as the primary venue for interaction among the materials research community and as an incubator for emerging areas of materials science. However, developments over the past decade, such as the growth of interdisciplinary materials science and the increasing interconnectedness of the global materials community, have prompted the MRS leadership to take a strategic look at the future of our meetings portfolio.

A major trend has been the evolution of materials science into a global endeavor with major centers of research spread throughout the Pacific Rim, India, Latin America, and Eastern Europe, and new materials communities emerging in Africa and the Middle East. This change is reflected in MRS membership, which is $40 \%$ non-U.S.

Additionally, there is a large and rapidly growing community of graduate students and postdoctoral fellows who would benefit greatly from the opportunity to participate in conferences like the MRS Fall and Spring Meetings, but often do not have the opportunity to do so. To be true to our vision of "... building a dynamic, interactive, worldwide community of materials researchers ... by providing a framework in which the materials disciplines can convene, collaborate, integrate and advocate," we must understand how MRS can provide this opportunity.

Another development is the dramatic way in which the web has changed global communication. Web-enabling companies, like Cisco, have discovered the power of virtual meetings in expanding the audience, amplifying the voice of speakers and exhibitors, and providing a vehicle for content delivery by those who cannot physically attend. When coupled with the recent expansion of MRS web capabilities and the other components of our communications portfolio, the potential to greatly expand the impact of our meetings through creative use of both web presence and print content becomes evident.

Further driving the global expansion of materials science is the recognition that the monumental challenges of energy and sustainability that the world must address are largely materials 


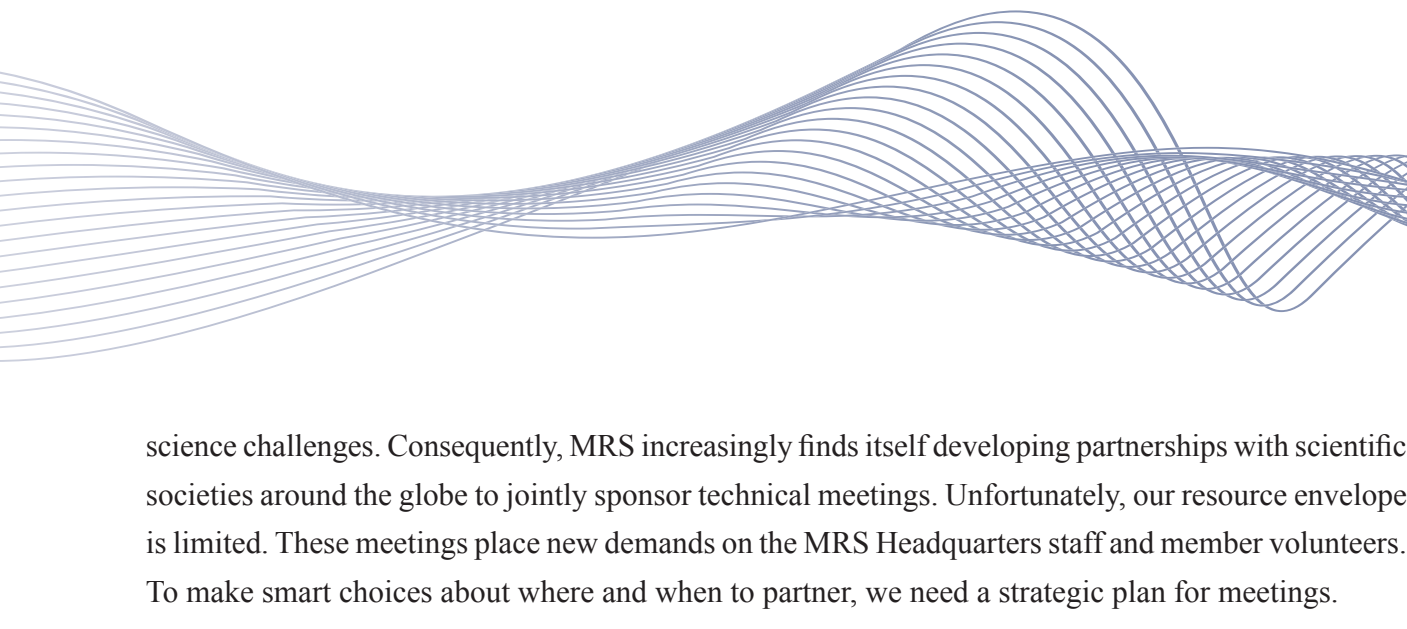

Beyond a consideration of technical content, the evolution of MRS from a meetings society to a "fullservice" professional society that materials researchers call home for much of their careers compels us to consider how we can best serve the full range of MRS missions. Over many years, MRS has greatly expanded its role in public outreach to make the field of materials science and engineering more accessible to the public and potentially more interesting as a career opportunity. We have also increased our interactions with government to advocate for the important role materials research has in addressing societal needs, which requires continued future investment. Communication by the Society is no longer directed strictly toward scientific professionals through technical meetings and publications, but rather toward all segments of society through a variety of venues. These activities are critical to the MRS strategic objective of ". . . advancing the sustainability of Materials Research" and are furthered by activities that take place at both the MRS meetings and others that we co-sponsor.

Over the last year, the Planning Committee of the Board of Directors has been leading a strategic meetings initiative to develop our 10-year vision for the future. This summer, four task forces-composed of operating committee members, Headquarters staff, and other experts - reported recommendations on the future technical landscape; opportunities for enhancing the Fall and Spring meetings; the important role of outreach, education, and advocacy at meetings; and our meetings partnership strategy. Following the annual Board of Directors Strategic Planning Meeting, which was held in conjunction with the International Materials Research Conference in August, we are clarifying how to best serve the materials community through our flagship meetings, other conferences, and workshops. We are also exploring ways to meaningfully integrate new technologies and win-win partnerships to advance the MRS mission.

The Planning Committee, chaired by Duane Dimos, will have a town hall discussion at the MRS Fall Meeting in Boston to share our current thinking and take input from the membership. We look forward to engaging all voices within MRS as we chart our course for the future.

In the meantime, if you have any questions, concerns, or ideas for what you believe represent important directions for MRS meetings, please send them to us at mtginfo@mrs.org.

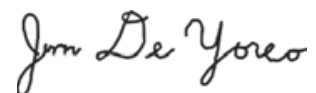

Jim De Yoreo

2011 MRS President

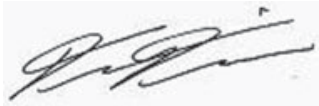

Duane Dimos

Chair, Planning Committee
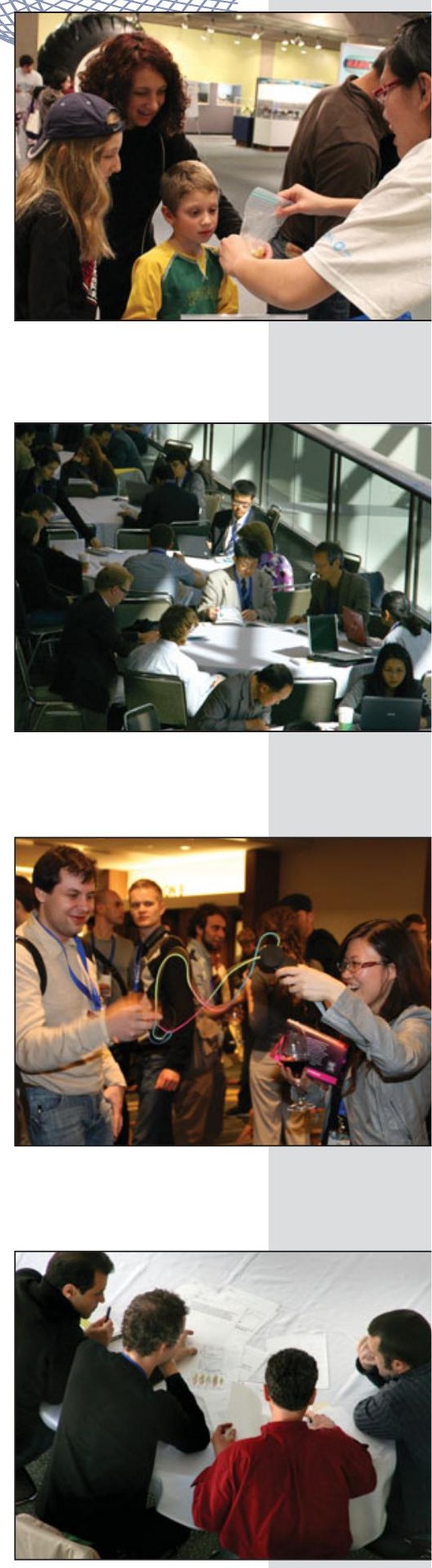